\title{
EKOSS: A Knowledge-User Centered Approach to Knowledge Sharing, Discovery, and Integration on the Semantic Web
}

\author{
Steven Kraines ${ }^{1}$, Weisen Guo $^{2,1}$, Brian Kemper ${ }^{1}$, and Yutaka Nakamura ${ }^{1}$ \\ ${ }^{1}$ Division of Project Coordination of the University of Tokyo, Tokyo 277-8568, Japan \\ \{sk, gws, bkemper, yutaka\}@cb.k.u-tokyo.ac.jp \\ 2 Inst. of Systems Eng. of Dalian University of Technology, Dalian 116024, China \\ guows@dlut.edu.cn
}

\begin{abstract}
The scientific enterprise depends on the effective transfer of knowledge from creator to user. Recently the rate of scientific knowledge production is overwhelming the ability for researchers to process it. Semantic web technologies may help to handle this vast amount of scientific knowledge. However, automatic computerized techniques that extract semantics from natural language text for use in matching with the requests of knowledge seekers achieve only mediocre results. Clearly, semantic descriptions of expert knowledge that are constructed by the knowledge creators themselves will be more accurate. We report an approach and software implementation of a knowledge sharing platform based on semantic web technologies, called EKOSS for expert knowledge ontology-based semantic search, that helps knowledge creators construct semantic descriptions of their knowledge. The EKOSS system enables knowledge creators to construct computer-interpretable semantically rich statements describing their knowledge with minimal effort and without any knowledge of semantic web technologies.
\end{abstract}

Keywords: semantic search, knowledge sharing, inference, ontology.

\section{Introduction}

The scientific enterprise has brought enormous wealth to society in the form of expert scientific knowledge. However, recently concern has been raised that knowledge is being produced in scientific research much faster than we can process it. Some writers have called this a "knowledge explosion", pointing to indicators such as an exponential increase in papers written in specific fields of scientific research [1, 2]. Semantic web technologies are being looked to as a potential approach for better handling this vast amount of scientific knowledge 3. We report an approach and software implementation, called EKOSS for expert knowledge ontology-based semantic search, to support knowledge sharing by using semantic web technologies. EKOSS takes the approach of enabling the knowledge creators to construct semantic descriptions of their knowledge that are better suited for computer-based processing and matching. In this paper, we 
describe the basic approach and architecture of the EKOSS system. An accompanying paper gives details on the EKOSS system implementation [28].

We have described the scientific enterprise as a knowledge cycle where knowledge generated by research scientists is continually returned to a global repository of scientific knowledge that forms the basis for further scientific research [5]. The cycle proceeds through stages of problem identification, experimental design, data analysis, and knowledge synthesis. At each stage of the process, the research scientist looks to the existing repository of scientific knowledge in the form of media such as papers and conference proceedings in order to discover previous research findings or hints that could help to achieve the goal of synthesizing useful new knowledge. Finally, the research scientist must input this new knowledge into the repository of scientific knowledge so that it is available to other research scientists for their research activities as well as to members of society for addressing various societal needs.

In the past, a diligent researcher could have read all of the papers in a particular field of scientific knowledge or at least scan abstracts of all potentially interesting papers. However, as the quantity of published results continues its exponential growth, researchers face the difficult problem of deciding which of the current research to invest time reading. Various techniques such as Google Scholar and the Web of Science can help researchers identify which scientific papers are most likely to be useful. However, despite remarkable progress in establishing computer-searchable electronic repositories of scientific papers and algorithms for extracting keywords automatically from those papers, the effectiveness of these techniques remains limited.

The fundamental problem is that scientific papers, in the way that they are published today, do not lend themselves well to computer-based techniques for matching papers with searches for knowledge. While scientific papers a century ago could be seen as a form of correspondence between people, writing a paper today can be compared to putting a message in a bottle and hoping that someone will find it on the Internet sea. Researchers cast a wide net using search engines such as Web of Science, but invariably they end up with many unrelated bottles, false positives, and they end up missing many important messages, false negatives. For example, we conducted a search on Google Scholar for a paper titled "a flexible model integration approach for evaluating tradeoffs between CO2 emissions and cost in solid oxide fuel cell-based building energy systems" that was published by one of us in the International Journal of Energy Research using the keywords "building" "distributed", "energy systems", "fuel cell", "gas turbine", and "electricity demand" that were contained in the paper's abstract. Only four papers in the top thirty hits had any reference to distributed energy systems in buildings that combine a fuel-cell with a gas turbine. The paper that we had wanted to find was the only paper that was actually about distributed energy systems contained in buildings that combine a fuel-cell with a gas turbine to supply electricity demand of buildings, and it ranked twenty-seventh out of thirty. 


\section{Using Semantics for More Accurate Searching}

By using tools and protocols developed for the realization of the Semantic Web, such as ontologies implemented in OWL-DL, statements with computerinterpretable semantics can be constructed. Logical inference and rule-based reasoning can be used to evaluate matches between such semantic statements, potentially resulting in a far more accurate mechanism for identifying what knowledge is most likely to be useful to a person who is seeking knowledge related to a particular condition, object, or design. For example, we could specify that the knowledge contained in the paper referred to above describes a study of a "building" containing a "distributed energy system" having as a part a "fuel cell" and as another part a "gas turbine" that is used to supply "electricity demand" of the "building". Using the predefined T-box structure of the ontology, a logical reasoning engine could reduce false negatives by knowing, for example, that "office building" is a type of "building" and that a "fuel cell" is a type of "energy device". Furthermore, by using an A-box to specify that both the "fuel cell" and the "gas turbine" are parts of the "energy system", which in turn is a part of the "building", we can reduce false positives. The role of semantics in reducing false positives and false negatives is illustrated in Fig. 1 ,

The effectiveness of this kind of semantic search is dependent on the accuracy and richness of the semantic statements. In particular, while natural language

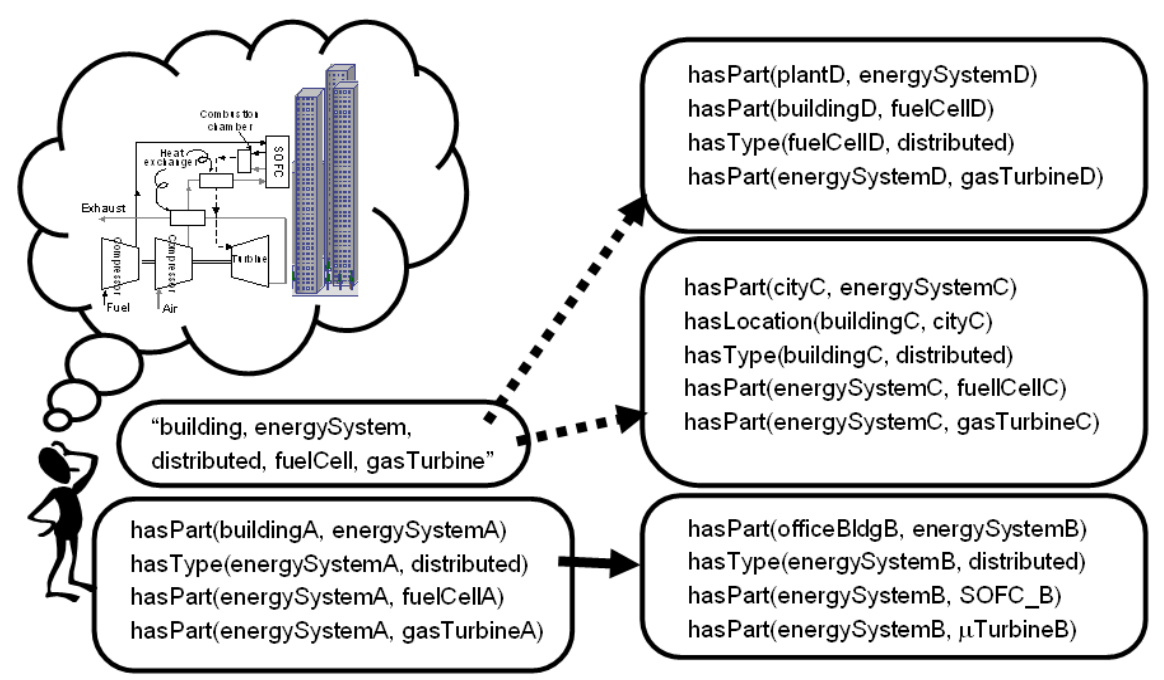

Fig. 1. Semantic matching versus keyword search. A researcher is looking for knowledge related to a "building" that has a part "energy system" with type "distributed", which in turn has parts "fuel cell" and "gas turbine". A simple keyword list matches with the wrong knowledge contents (broken lines). Only the semantic description with declared relationships between the instances of the keywords matches with the correct knowledge contents (solid line). 
processing (NLP) techniques can help to extract useful semantics from scientific papers [6], [7, [8], even the best NLP methods still do not perform well in terms of precision and recall of scientific papers [9, 10].

Semantic statements made by the researchers who created the knowledge could be expected to be more accurate. We argue that researchers would be willing to make at least a small effort to publish their knowledge in a particular format if that format could be guaranteed to be significantly more effective at reaching the people who can most benefit from the knowledge. This is analogous to attaching a transmitter that sends out a signal "advertising" the content of the bottle. Authors of papers and conference abstracts do this when they choose keywords to attach to their manuscript that they feel most accurately represent the knowledge contained, and using a controlled vocabulary for keywords helps make the transmission more clear. We believe that, with only a small additional effort, researchers could attach far more effective transmissions to their papers through the use of semantic statements based on formal ontologies. Alternatively, reviewers or editors could attach semantic descriptions of the papers based on their objective understanding of the main messages in the paper. The key requirements in our approach are that the process of authoring the semantic descriptions be decentralized and that the authoring process not require any special expertise on knowledge engineering or semantic technologies.

We have developed a prototype web-based system, called EKOSS for Expert Knowledge Ontology-based Semantic Search, to support this kind of semantic markup of both scientific papers and other knowledge resources that describe some expert knowledge and search requests for scientific knowledge related to specific conditions and/or problems. We use ontologies based on description logics to provide simplified languages in specific domains of knowledge that can be reasoned against using DL reasoning software. The EKOSS system lets users make both semantic descriptions of knowledge and semantic queries to express search requests for that domain. We also support the reverse: that is semantic queries to express conditions that must be filled for particular knowledge to be applicable and semantic descriptions of the particular conditions for which knowledge is being sought. We use the RacerPro reasoner to evaluate the matches between knowledge descriptions and search requests as well as to evaluate the consistency of semantic descriptions and queries for both knowledge and searches [11. The EKOSS system supports the use of multiple distinct ontologies. A set of administrator tools are provided for loading new ontologies and modifying existing ones, and we are developing user interfaces for modifying the existing ontologies as described in the last section of this paper.

\section{The Role of EKOSS in Scientific Knowledge Sharing}

The EKOSS system is being developed as a part of a four level architecture for scientific knowledge sharing, discovery, and integration that we have described in previous publications [5, 13, [12. Briefly, the four levels of the architecture framework, shown in Fig. 2, are as follows. Knowledge resources, including 
scientific papers, databases and simulation models, created by knowledge experts around the world form the base level of the framework. Tools are provided at the second level to each knowledge expert in order to publish semantic descriptions of that person's expert knowledge. At the third level, software agents representing each of the knowledge experts communicate to identify potentially useful knowledge sharing opportunities based on the semantic descriptions constructed at the second level [13, [14. The fourth level holds knowledge integration technologies, such as the semantic distance matrix generation tools that we describe at the end of this paper and the distributed object-based modeling environment DOME being developed at the CAD laboratory of the Massachusetts Institute of Technology. DOME can be used to integrate the knowledge discovered at the third level over the Internet in order to rapidly synthesize new integrated knowledge through scenario analyses and optimization [15].

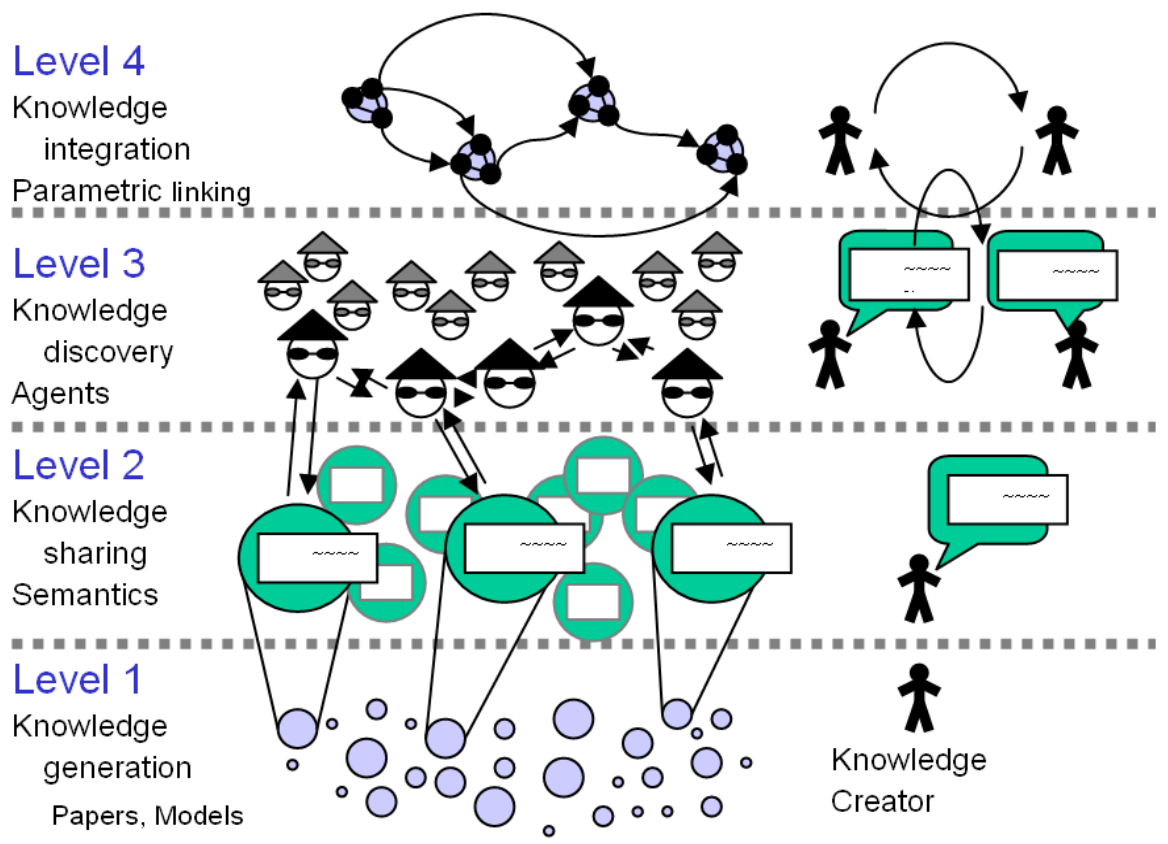

Fig. 2. The four level architecture for knowledge sharing, discovery and integration proposed by the authors

The EKOSS system is intended to implement the second level of this framework. Specifically, the EKOSS system provides a web-accessible knowledge sharing platform with the following features:

- an ontology browser for examining the available ontologies and supporting dialogue concerning ontology development,

- a personal repository of Knowledge Projects that contain semantic descriptions and queries for representing specific expert knowledge of a user, 
- a personal repository of Search Projects that contain semantic descriptions and queries that represent knowledge interests or requirements of a user,

- a semantic search engine based on the RacerPro DL reasoner with extensions for rule-based reasoning and datatype reasoning.

EKOSS Knowledge Projects and Search Projects have essentially the same structure. Each project can contain one or more semantic descriptions that represent the details of that project, i.e. the knowledge contained or the conditions behind the search. Projects also can contain several generalized semantic queries to represent the conditions that should be filled in order to establish a match. Often, the Search Projects will contain semantic queries, such as "find me all knowledge projects that mention buildings that contain fuel cells", and the Knowledge Projects will contain semantic descriptions, such as "this is knowledge about a office building that contains an energy system that in turn contains a polymer electrolyte fuel cell". However, the reverse is also useful. For example, a semantic query can be used to specify a particular condition that must hold for the knowledge represented by a Knowledge Project to be applicable: "this knowledge is applicable to situations where there exists a building whose energy demand is supplied by a local energy device". Search Projects can then make use of semantic descriptions to describe the particular conditions for which knowledge is being sought: "we are designing an office building with a microturbine as an energy supply device".

We give a simple example here of creating a semantic description for the matching knowledge shown in Fig. 1, A researcher seeking to share her knowledge regarding buildings with energy systems that have fuel cells and gas turbines logs in to her local EKOSS server and creates a new knowledge project. After entering a name and other information for the project, she clicks a button to add a semantic description to the project and selects one of the ontologies available on the EKOSS server to create an A-box describing the object of her knowledge. The EKOSS ontology browser is displayed, and the researcher navigates the subsumption taxonomy of the selected ontology to find the concept for building. After navigating the subsumption tree to the class in the ontology that best represents the concept that she wants, she presses the "Add" button to create an instance of the selected class, "building", in her A-box and optionally adds a text label to the instance.

The researcher can repeat the process above to add another instance or use the "Add Property" interface to add an object or datatype property to an existing instance. Selecting "Add Property" displays a tree of properties that can take "building" as a domain class (Fig. 3). The researcher wants to indicate that the building has as a part an energy system with a fuel cell and a gas turbine, so she finds the property "has_part", which is a subproperty of "composition_of_individual". The "Add Property" interface shows a subsumption tree of the classes that can be ranges of "has_part". The researcher finds the class "energy_system" and sets it to be the range of "has_part". The EKOSS system creates an instance of "energy_system" and again lets the user label the instance 
if desired. The user continues to create instances and properties until the A-box for the knowledge description is completed. An interactive directed graph of the A-box can be displayed by selecting "graph view" (Fig. 4).

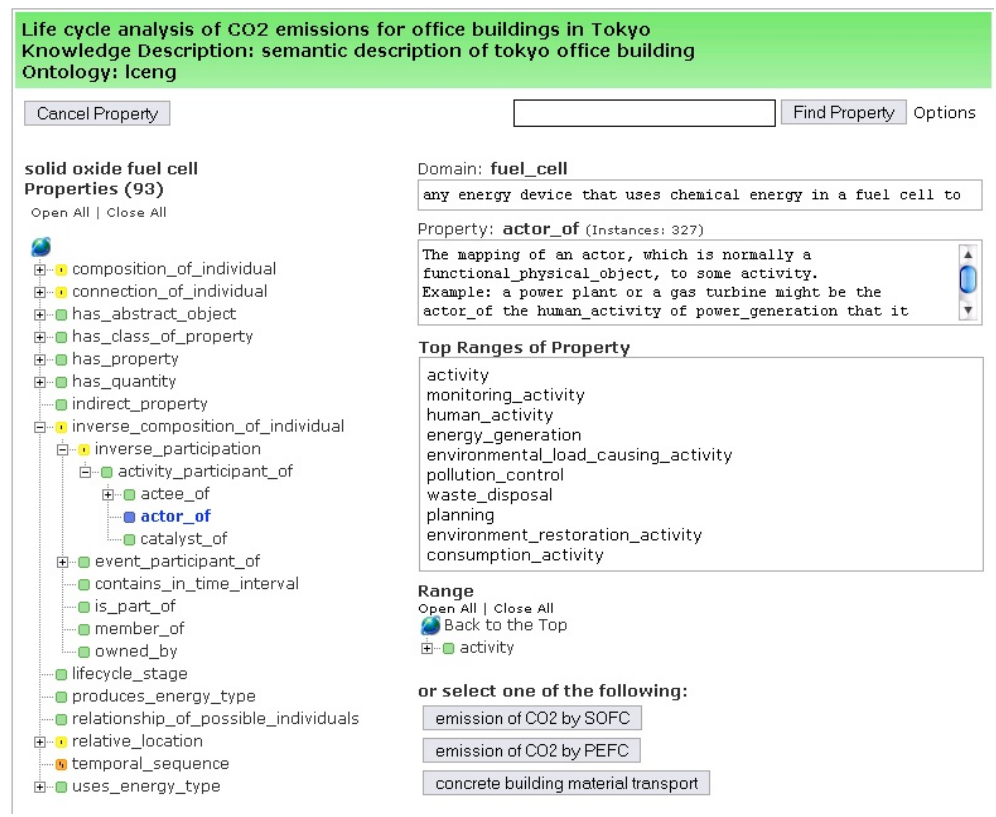

Fig. 3. The "add property" page for adding properties to an instance of the class "fuel_cell"

Semantic descriptions and semantic queries are completely interchangeable, that is a semantic description of one particular project can be declared to be a semantic query or description of another Knowledge or Search Project. Moreover, a Knowledge Project can function as a Search Project and vice versa. This feature of EKOSS both enhances its effectiveness and reduces the learning curve. Each Knowledge and Search Project serves as a node on a directed graph of knowledge, with links between project nodes directed from the queries of the origin project to the descriptions of the destination project (Fig. 55). For example, a directed link from project A to project B means that the abstraction of the knowledge (or request for knowledge) represented by queries in project $\mathrm{A}$ is matched, at least in part, by the detailed semantic representation of the knowledge (or search conditions) in project $B$.

Multiplicity of semantic queries has a special meaning - by creating multiple versions, that is subsets, of a template query, it is possible to evaluate partial matches and special conditions for a match between that project and the semantic description of another project (see 4] for more details). For each semantic query in a Knowledge or Search Project, the project creator can set a matching weight value and a "match type" flag. The "match type" flag specifies whether 


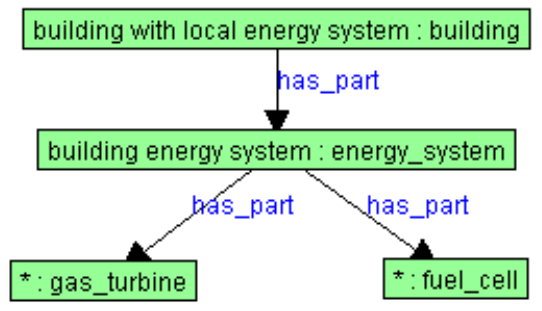

Fig. 4. Graph view of the completed semantic knowledge query. Boxes indicate instances, and arrows show the property connections. The actual instance class is shown to the right of the colon; the user defined instance label is shown to the left of the colon.

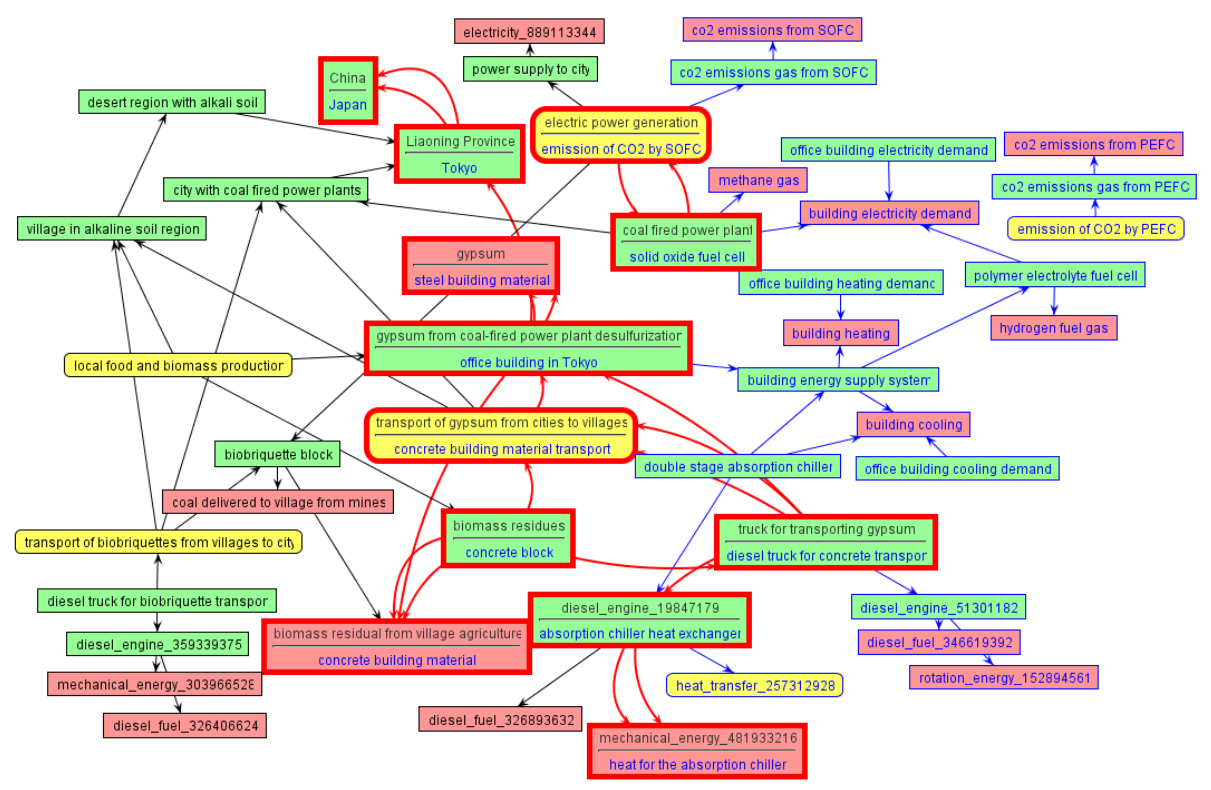

Fig. 5. Example of a match between two semantic knowledge description A-boxes. The instances in A-box used as the search condition are shown with black labels, and the instances in the A-box used as the matching knowledge description are shown with blue labels. The color of the instances indicates the type of instance. Arrows show the properties in the A-boxes connecting the different instances. The matching instances are shown with thick red borders.

(a) all of the instances in the query must bind with instances in the description in order to score a successful match and contribute the weight of the query to the overall match score (the default behavior) or (b) at least one of the instances must not bind with any instances in the description in order to score the successful match. For example, we can represent the following search condition: "I am 
looking for a building that should contain an energy system, preferably contains a fuel cell, must not contain a diesel engine, and should not contain any kind of engine" using the four queries and settings shown in Table 1 .

Table 1. Search queries, match types, and weights for the example given in the text

\begin{tabular}{lll}
\hline Search Query & Match Type & Weight \\
\hline building physically_contains energy_system & Must Match & 1 \\
building physically_contains fuel_cell & Must Match & -2 \\
building physically_contains diesel_engine & Must Not Match & 5 \\
building physically_contains engine & Must Not Match & 1 \\
\hline
\end{tabular}

\section{Approaches for Constructing Semantic Descriptions Based on the EKOSS Experience}

We are currently employing over 30 students from both undergraduate and graduate courses in fields of environmental technologies and life sciences at the University of Tokyo to create semantic descriptions for the EKOSS server that we have deployed at http://www.ekoss.org. From our own experiences in creating semantic descriptions of knowledge and search conditions for the EKOSS system together with student feedback, we have found that three approaches are particularly effective. The first approach to constructing a semantic description is to begin by making a list of about 10 to 20 keywords that describe the knowledge or search conditions you are considering. Then, for each of the keywords, use the ontology browser function of the "Add Instance" tool to find the class in the ontology that best expresses that keyword. After you have created instances of the ontology classes for each of the keywords, use the "Add Connection" tool to determine the ontology properties that best express the binary relationships between the instances you have created. Finally, you can add additional instances and properties to describe particular attributes of the keywords. A typical example is a keyword that is really best expressed by two classes in the ontology, such as "building energy system". This keyword could be expressed by the A-box ("energy_systemA" "is_part_of" "buildingB").

The second approach that we have found to be effective for constructing semantic descriptions is to begin with a single instance of an ontology class that best describes the object of the knowledge or search. Properties can then be added to connect to other classes in the ontology in order to indicate the attributes that make the object special. For example, knowledge about an office building located in Tokyo that contains a fuel cell used for energy supply could be described by first creating an instance of the class "office_bldg" and then adding the property "physically_contains" pointing to an instance of "fuel_cell", "has_location" pointing to an instance of "city" labeled "Tokyo", and so on.

The third approach that we have used is to start with a relatively clear image of what the graph of instances and properties should look like and then build 


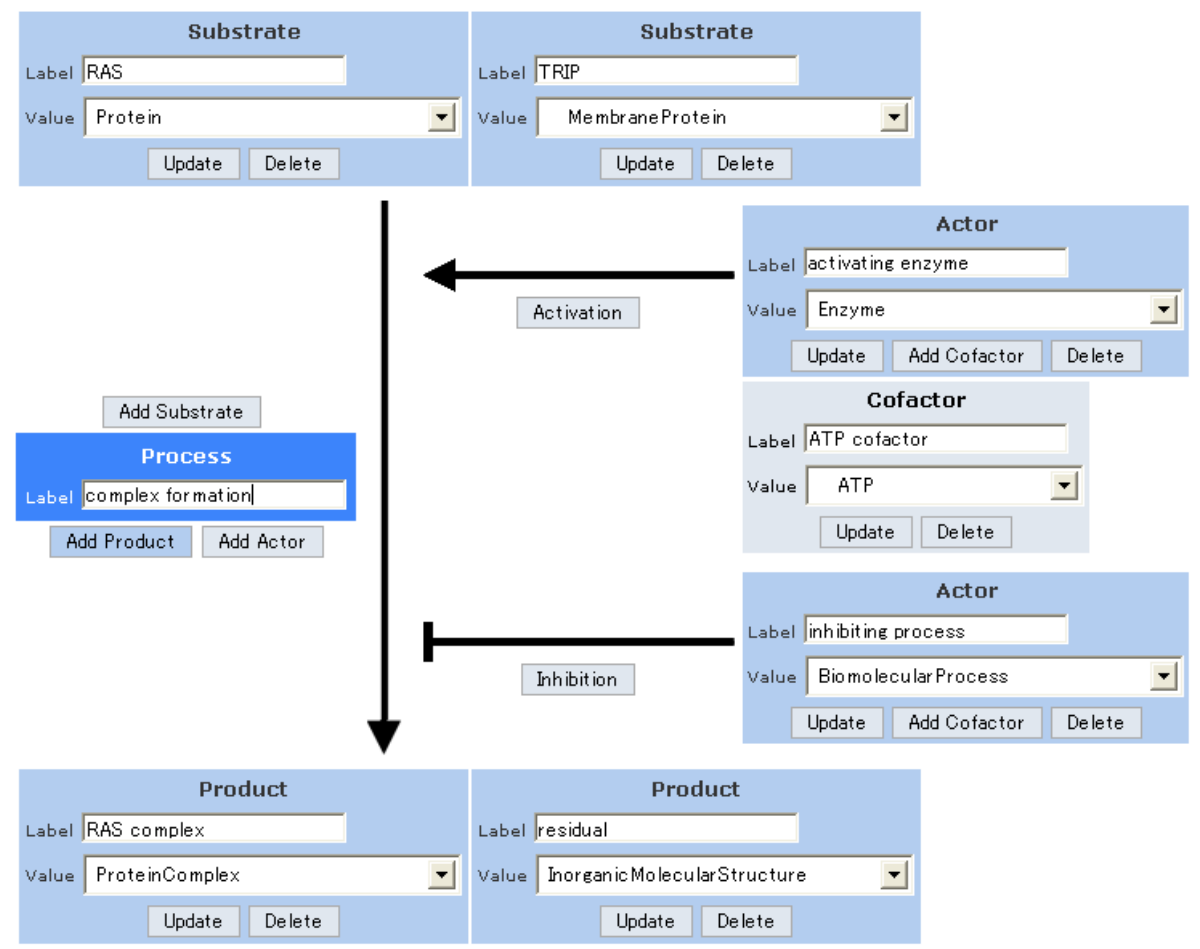

Fig. 6. The biology interface wizard for constructing descriptions of biomolecular processes

that graph. We have found that several common patterns appear in semantic descriptions from particular knowledge domains. For example, in the field of metabolic pathways, much of the knowledge centers on a particular biomolecular process, such as phosphorylation, that has one or more substrates and one or more products. Furthermore, most processes in cellular biology have one or more "actors" that either activate or inhibit the process. This simple model of metabolic pathway knowledge can be expressed by a basic template for semantic descriptions of that knowledge, as shown in Fig. 6. We are developing a number of such templates for commonly occurring patterns in knowledge from different domains that are supported by our system, as described in the section "Future Directions".

\section{Comparison of EKOSS with Existing Systems}

There are a number of web platforms that have been developed to make use of semantic web technologies for supporting knowledge sharing. Each platform uses different degrees of structure in the computer-interpretable semantics, provides different types of services to its users, and offers different features for assisting 
users in the process of adding semantics to their knowledge resources. Here, we compare some of these semantic knowledge sharing systems with EKOSS.

Mangrove, developed at the University of Washington, aims to provide a semantic-enabled knowledge sharing platform for communities of "non-technical" people. The philosophy of the Mangrove Project is that non-technical users could be enticed to semantically annotate their web pages if they were provided with useful services that gave them a sense of "instant gratification" [16]. The semantics used by the Mangrove system are therefore necessarily simplistic, and there is no underlying knowledge model or ontology for supporting logical reasoning. Although a proprietary reasoning engine is mentioned, it is unclear what reasoning capabilities are supported.

Dspace has been developed through the collaboration of the W3C, the MIT libraries, and the MIT CSAIL (Computer Science and Artificial Intelligence Laboratory) as a digital library that uses semantic tags to help with cataloging and retrieval 17. DSpace is being augmented by the SIMILE (Semantic Interoperability of Metadata and Information in unLike Environments) project to support arbitrary metadata schemata in the form of RDF markup [18. However, while EKOSS provides tools for using DL ontologies to create semantic A-boxes that describe knowledge resources in a way that can be reasoned against with DL reasoners for more accurate matching and retrieval, the semantics supported by Dspace with SIMILE is limited to RDFS so that only reasoning based on class subsumption is possible.

Magpie is a product of the Advanced Knowledge Technologies project that takes the form of a browser plugin providing users with tools for displaying terms in web pages that are found to match with terms from the ontology loaded in the system [22]. As the goal is to provide semantic web services with near-zero overhead costs, speed and minimization of user input requirements are emphasized. The EKOSS system, on the other hand, aims to empower knowledge creators interested in sharing their knowledge with the capacity to create semantic descriptions of that knowledge themselves with only a small amount of effort, trading off a small increase in overhead costs for the potential benefits from the greatly increased semantic richness.

SMORE (Semantic Markup, Ontology, and RDF Editor) is a set of tools created by MINDSWAP and integrated with the SWOOP ontology browser and the pellet OWL-DL reasoner that are intended to help users annotate their own web documents in OWL using ontologies on the web, without requiring the users to know OWL terms and syntax 24]. SMORE also provides limited capabilities for creating new ontologies and modifying existing ontologies to incorporate terms from the user's web documents. In this sense, the SMORE tools are designed for ontology building and annotation of web documents rather than actually describing knowledge resources with computer interpretable semantics.

CONFOTO supports the semantic annotation and browsing of conference photographs with RDF tags that are based on keyword-based classifications or folksonomies [19]. Folksonomies have been hypothesized to be easier for users to use in creating metadata than formal ontologies. However, we believe that 
through the tools and interfaces provided by the EKOSS system, a significant number of users particularly in the sciences would be willing to make the investment in time and effort to create semantic descriptions that would be far more useful for semantic analysis due to their solid foundation in formal ontologies.

Other related technologies include Cyc and other ontologies that are intended to function as centralized knowledge bases 21, the SEKT (Semantically-Enabled Knowledge Technologies) EU initiative that aims to realize a European Knowledge Society through development of core semantic technologies for knowledge management based on the KAON reasoner [20], the Tucana Knowledge Discovery Platform and its open source versions Kowari and Mulgara that target semantic databases and knowledge discovery platforms for enterprises [23, the SWOOGLE web search engine for searching and analyzing web documents having semantic markup [25], the Ont-O-Mat implementation of the CREAM framework for helping web page authors create semantic annotations in the form of relational metadata [27, and the web-based Annotea system for supporting the creating and sharing of RDF annotations [26].

\section{$6 \quad$ Future Directions}

In future research work, we plan to continue the development of the EKOSS system along three paths. First, we will develop domain specific wizards for creating semantic descriptions. These wizards will include 1) pictorial visualizations of ontology for helping users to identify the class in the ontology that best describes a concept that they want to include in an A-box and 2) template wizards for supporting construction of A-boxes for common semantic patterns in particular domains. An example of the second type of wizard was shown in Fig. 6,

We will also extend the functionality of the ontology browser in order to support user dialogue about the classes and structures of the ontologies available in the system, including examples of uses, suggestions for modifications to the ontologies, and addition of new classes. In doing so, we intend to offer expert users the opportunity to "bootstrap" the process of development and refinement of the ontologies on a particular EKOSS server. Furthermore, we will investigate the effectiveness of techniques for ontology translation and alignment based on the A-boxes that are created using the different domain ontologies supported by the EKOSS system.

Finally, on the semantic matching side, as we begin to accumulate a sufficient number of Knowledge Projects in the EKOSS system, we will develop methods for determining the semantic distance between pairs of semantic descriptions representing the Knowledge Projects. One approach that we are using is to create tools for automatically generating interesting subsets of the relatively large semantic description A-boxes and using those subsets as queries for matching with the semantic descriptions of other Knowledge Projects. The result is an indication of the degree of overlap between the two semantic descriptions (Fig. (5). We can use these measured distances between sets of Knowledge Projects to construct different semantic distance matrices depending on the conditions used 
for generating the queries from the semantic description of the first Knowledge Project. These semantic distance matrices can be used for a variety of knowledge integration applications. For example, we are developing graphic interface tools for visualizing the overall knowledge network that is represented by these semantic distance matrices as two dimensional knowledge maps or as three dimensional visualizations of Knowledge Project distributions around a particular project.

Acknowledgement. We gratefully acknowledge contributions and advice from Rafael Batres, Hiroshi Komiyama, Toshihisa Takagi, and David Wallace. Funding support was provided by the Knowledge Failure Database project at the Japan Science and Technology Agency.

\section{References}

1. Beasley, S.W.: The value of medical publications: 'To read them would ... burden the memory to no useful purpose'. Australian and New Zealand Journal of Surgery 70 (2000) 870-874

2. Ziman, J. M.: The proliferation of scientific literature: a natural process. Science 208 (2004) 369-371

3. Berners-Lee, T., Hendler, J.: Publishing on the Semantic Web. Nature 410 (2001), 1023-1024

4. Guo, W., Kraines, S.B.: Achieving Scalability of Semantic Searching and Matching based on OWL-DL, Rules and Datatype Property Reasoning. Submitted to SSWS2006

5. Kraines, S.B., Kemper, B.E., Wallace, D.R., Komiyama, H.: Scientific knowledge sharing through ontologies and computational models. Submitted to Communications of the ACM

6. Craven, M., Kumlien, J.: Constructing biological knowledge bases by extracting information from text sources. Proceedings of the 7th Intl Conf. on Intelligent Systems for Molecular Biology (ISMB-99) (1999)

7. Huang, M., Zhu, X., Hao, Y., Payan, D.G., Qu, K., Li, M.: Discovering patterns to extract protein-protein interactions from full texts. Bioinformatics 20 (2004) 3604-3612

8. Rzhetsky, A., Koike, T., Kalachikov, S., Gomez, S.M., Krauthammer, M., Kaplan, S.H., Kra, P., Russo, J.J., Friedman, C.: A knowledge model for analysis and simulation of regulatory networks. Bioinformatics Ontology 16 (2000) 1120-1128

9. Abbott, R.: Subjectivity as a concern for information science: a Popperian perspective. Journal of Information Science 30 (2004) 95-106

10. Swanson, D.R.: Medical literature as a potential source of new knowledge. Bull. Med. Libr. Assoc. 78 (1990) 29-37

11. Racer Systems GmbH \& Co. KG.: World Wide Web site http://www.racersystems.com

12. Kraines S.B., Batres, R., Koyama, M., Wallace, D.R., Komiyama, H.: InternetBased Integrated Environmental Assessment: Using Ontologies to Share Computational Models. J. Industrial Ecology 9 (2005) 31-50

13. Kraines S.B., Wolowski, V., Koyama, M., Kemper, B.E., Muraki, R., Batres, R.: A semantic search engine for discovering engineering models using ontologies and agents. J. Industrial Ecology. In press 
14. Guo, W., Kraines, S.B.: Knowledge sharing on a multi-agent reputation-based trust network. In preparation

15. Wallace, D.R., Abrahamson, S., Senin, N., Sferro, P.: Integrated design in a service marketplace. Computer-aided Design 32 (2000), 97-107

16. McDowell, L., Etzioni, O., Gribble, S., Halevy, A., Levy, H., Pentney, W., Verma, D., Vlasseva, S.: Mangrove: enticing ordinary people onto the semantic web via instant gratification. In Second International Semantic Web Conference (ISWC 2003), October 2003

17. Dspace website: http://www.dspace.org/

18. Mazzocchi, S., Garland, S., Lee, R.: SIMILE: Practical Metadata for the Semantic Web. XML.com, O'Reilly Media, Inc. January 26, 2005, http://www.xml.com/pub/a/2005/01/26/simile.html

19. Nowack, B.: CONFOTO: A semantic browsing and annotation service for conference photos. In Fourth International Semantic Web Conference (ISWC 2005) Y. Gil et al. (Eds.), LNCS 3729, pp. 1067-1070, 2005

20. SEKT website: http://www.sekt-project.org/

21. cyc website: http://www.opencyc.org/

22. Dzbor, M., Motta, E., Domingue, J.: Opening up magpie via semantic services. In Third International Semantic Web Conference (ISWC 2004), LNCS 3298, pp. 635-649, 2004

23. tucana website: http://tucana.es.northropgrumman.com/

24. Kalyanpur, A., Parsia, B., Hendler, J., Golbeck, J.: SMORE - semantic markup, ontology, and RDF editor. http://www.mindswap.org/papers/SMORE.pdf

25. swoogle website: http://swoogle.umbc.edu

26. Kahan, J., Koivunen, M.: Annotea: an open RDF infrastructure for shared web annotations. WWW2001, Proceedings of the Tenth International World Wide Web Conference. Hong Kong, May 2001.

27. Handschuh, S., Staab, S.: Authoring and annotation of web pages in CREAM. WWW2002, Proceedings of the Eleventh International World Wide Web Conference. Honolulu, Hawaii, USA, May 2002.

28. Kraines, S.B., Guo, W., Kemper, B., Nakamura, Y.: A semantic web application for expert knowledge sharing, discovery, and integration. ISWC2006. In press 